\title{
Interval-valued intuitionistic fuzzy generalized nets
}

\author{
Krassimir Atanassov ${ }^{1}$, Dafina Zoteva ${ }^{1}$ and Nora Angelova ${ }^{2}$ \\ ${ }^{1}$ Department of Bioinformatics and Mathematical Modelling \\ Institute of Biophysics and Biomedical Engineering \\ Bulgarian Academy of Sciences \\ 105 Acad. Georgi Bonchev Str., Sofia 1113, Bulgaria \\ e-mails: krat@bas.bg, dafy.zoteva@gmail .com \\ 2 Faculty of Mathematics and Informatics \\ Sofia University "St. Kliment Ohridski” \\ 5 Blvd "James Bourchier", Sofia 1164 Bulgaria \\ e-mail: noraa@fmi.uni-sofia.bg
}

Received: 28 March 2018

Accepted: 7 June 2018

\begin{abstract}
New extensions of the concepts of Intuitionistic Fuzzy Generalized Nets of Type 1 (IFGN1) and of Type 3 (IFGN3), namely Interval-Valued Intuitionistic Fuzzy Generalized Nets (IVIFGN) of First Type and IVIFGN of Third Type are introduced in the present paper. The general algorithms for transition and generalized net (GN) functioning in IVFGN of First Type are presented. It is proved that the IVIFGN1s and IVIFGN3s are conservative extensions of the GNs, i.e., their functioning and results of their works can be described by standard GNs.
\end{abstract}

Keywords: Generalized nets, Intuitionistic fuzzy generalized nets, Interval-valued intuitionistic fuzzy generalized nets.

2010 Mathematics Subject Classification: 03E72, 68Q85.

\section{Introduction}

The concept of a Generalized Net (GN, see $[1,4,6])$ was introduced in 1982 as an extension of the Petri nets and their extensions and modifications. 
In 1985, in [2], two of the GN-extensions were introduced and in 2001 in [11], these extensions were extended again. In [2] were defined the Intuitionistic Fuzzy GNs of first and second types (IFGN1s and IFGN2s) and in [11] - the Intuitionistic Fuzzy GNs of third and fourth types (IFGN3s and IFGN4s).

The IFGNs are based on the intuitionistic fuzzy sets theory $[5,7]$ and all notations in the paper, related to intuitionistic fuzziness are defined in these two books.

Here, we mention only that the Interval-Valued Intuitionistic Fuzzy Pair (IVIFP) is an object with the form $\langle M, N\rangle$, where $M, N \subseteq[0,1]$ are closed sets, $M=[\inf M, \sup M], N=[\inf N, \sup N]$ and $\sup M+\sup N \leq 1$, that is used as an evaluation of an object or a process and which components $(M$ and $N)$ are interpreted as intervals of degrees of membership and non-membership, or intervals of degrees of validity and non-validity, or intervals of degree of correctness and noncorrectness, etc. One of the basic geometrical interpretations of an IVIFP is shown on Figure 1.

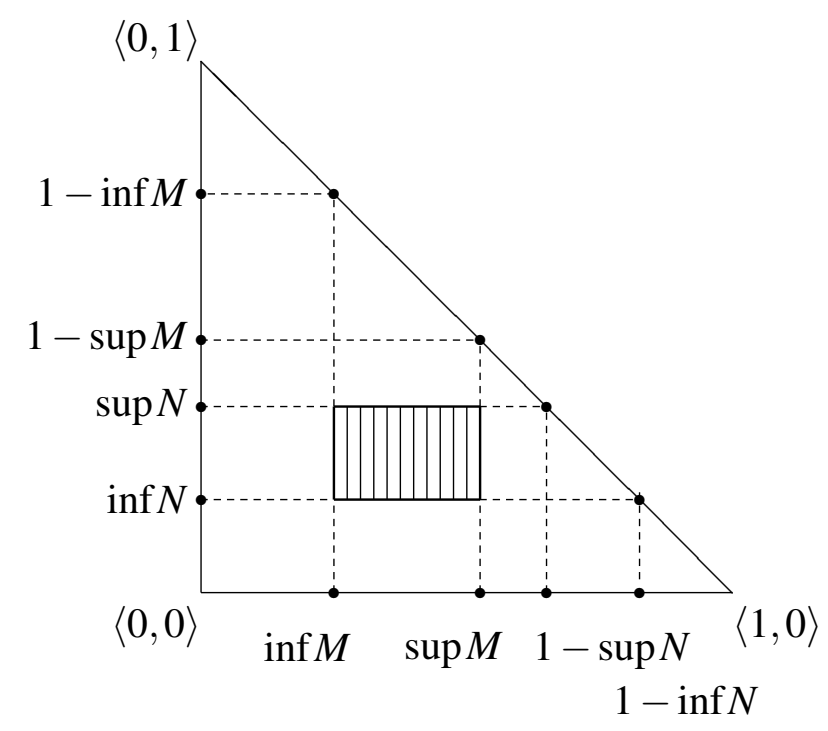

Figure 1. Geometrical interpretation of an IVIFP

It is suitable to mention that the logical true and false are represented, respectively, by the IVIFP $\langle[1,1],[0,0]\rangle$ and $\langle[0,0],[1,1]\rangle$. Obviously, these values correspond to the intuitionistic fuzzy points $\langle 1,0\rangle$ and $\langle 0,1\rangle$.

\section{Interval-valued intuitionistic fuzzy generalized nets of first type}

In the present paper we will extend the IFGN1s and IFGN3s. Similarly to Petri nets, the GNs in all their modifications contain transitions, places and tokens. The GN-place is depicted by circle, and the GN-transition is depicted on Figure 2. A GN, as well as the other nets, contains tokens which transfer from place to place. But unlike all other nets, every GN-token enters the net with an initial characteristic and it receives new characteristics during each transfer. Every place has at most one arc entering and one arc exiting. The places with no entering arcs are called input 
places of the net and those without exiting arcs are output places of the net. The places situated to the left of the transition are the transition's input places while the rest are the transition's output places.

Each transition has at least one input and one output place. When tokens enter the input places of a transition, it becomes potentially fired (activated) and at the moment of their transfer towards the transition's output places, it is fired. The transition is activated at a given time-moment and then becomes active again at some subsequent time-moment which is predetermined.

The first basic difference between GNs and the ordinary Petri nets is the "place - transition" relation. Here, the transitions are objects of a more complex nature. Every transition contains $m$ input and $n$ output places, where $m, n \geq 1$.

Definition 1. Every IVIFGN1-transition is given by a seven-tuple (see Figure 2):

$$
Z=\left\langle L^{\prime}, L^{\prime \prime} t_{1}, t_{2}, r, M, \square\right\rangle
$$

where

a) $L^{\prime}$ and $L^{\prime \prime}$ are finite, non-empty sets of places (the transition's input and output places, respectively);

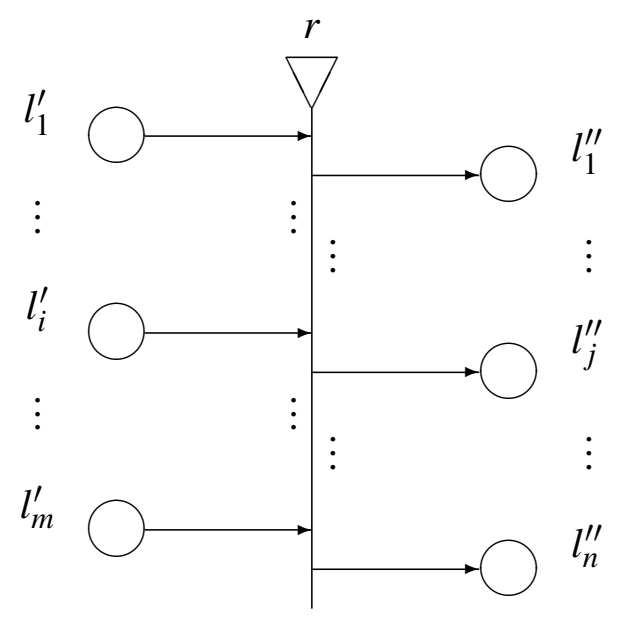

Figure 2. A GN-transition

b) $t_{1}$ is the current time-moment of the transition's firing;

c) $t_{2}$ is the current value of the duration of its activity;

d) $r$ is the transition's condition determining the tokens which will transfer from the transition's inputs to its outputs; it has the form of an Index matrix (IM, see [3, 8]):

$$
r \begin{array}{c|c} 
& l_{1}^{\prime \prime} \ldots l_{j}^{\prime \prime} \ldots l_{n}^{\prime \prime} \\
\hline l_{1}^{\prime} & \\
\vdots & r_{i, j} \\
l_{i}^{\prime} & \left(r_{i, j}-\text { predicates }\right) \\
\vdots & (1 \leq i \leq m, 1 \leq j \leq n) \\
l_{m}^{\prime} &
\end{array}
$$


where $r_{i, j}$ denotes the element of the IM, which corresponds to the $i$-th input and $j$-th output places. These elements are predicates and when the truth value of the $(i, j)$-th element $f\left(r_{i, j}\right)=$ $\left\langle M_{i, j}, N_{i, j}\right\rangle$ is valid according to one of the 15 conditions given below, the token from the $i$-th input place can be transferred to the $j$-th output place; otherwise, this is not possible. Here $M_{i, j}, N_{i, j} \subseteq[0,1]$ and $\sup M_{i, j}+\sup N_{i, j} \leq 1$ and function $f$ is defined in point e) of Definition 2 . The conditions for interval-valued intuitionistic fuzzy validity of the predicates are the following:

- $(\mathbf{C 1}) \inf M=1$ (therefore $\sup M=1$ and $\inf N=\sup N=0)$,

- (C2) $\sup M=1($ therefore $\inf N=\sup N=0)$,

- (C3) $\frac{1}{2}<\inf M \leq 1$ and $\sup N=0$,

- (C4) $\inf M>\frac{1}{2}>\sup N$,

- (C5) $\inf M \geq \frac{1}{2} \geq \sup N$,

- (C6) $\sup M>\frac{1}{2}>\sup N$,

- (C7) $\sup M \geq \frac{1}{2} \geq \sup N$,

- (C8) inf $M>\sup N$,

- (C9) $\inf M \geq \sup N$,

- (C10) $\sup M>\sup N$,

- (C11) $\sup M \geq \sup N$,

- (C12) $\sup M>0$,

- (C13) $\inf M>0$,

- (C14) $\sup N<1$,

- (C15) $\inf N<1$.

Therefore, the tokens's transfer from $i$-th input to $j$-th output places is possible for a fixed criterion, when the components of $\left\langle M_{i, j}, N_{i, j}\right\rangle$ satisfy the respective criterion;

e) $M$ is an IM of the capacities of transition's arcs:

$$
M=\begin{array}{c|c} 
& l_{1}^{\prime \prime} \ldots l_{j}^{\prime \prime} \ldots l_{n}^{\prime \prime} \\
\hline l_{1}^{\prime} & \\
\vdots & M_{i, j} \\
l_{i}^{\prime} & \left(M_{i, j} \geq 0-\text { natural numbers }\right) \\
\vdots & (1 \leq i \leq m, 1 \leq j \leq n) \\
l_{m}^{\prime} &
\end{array}
$$


f) $\square$ is an object whose form is similar to a propositional formula. It may contain symbols among the labels of the transition's input places as variables, and the Boolean operations $\wedge$ and $\vee$. We assign the following semantics to this formula:

$\wedge\left(l_{i_{1}}, l_{i_{2}}, \ldots, l_{i_{u}}\right)$ - every place $l_{i_{1}}, l_{i_{2}}, \ldots, l_{i_{u}}$ must contain at least one token, $\vee\left(l_{i_{1}}, l_{i_{2}}, \ldots, l_{i_{u}}\right)$ - there must be at least one token in all places $l_{i_{1}}, l_{i_{2}}, \ldots, l_{i_{u}}$ where $\left\{l_{i_{1}}, l_{i_{2}}, \ldots, l_{i_{u}}\right\} \subset L^{\prime}$.

When the value of a type, evaluated as a Boolean expression, is true, the transition can become active, otherwise it cannot.

Definition 2. The ordered four-tuple

$$
E=\left\langle\left\langle A, \pi_{A}, \pi_{L}, c, f, \theta_{1}, \theta_{2}\right\rangle,\left\langle K, \pi_{K}, \theta_{K}\right\rangle,\left\langle T, t^{o}, t^{*}\right\rangle,\langle X, \Phi, b\rangle\right\rangle
$$

is called an Interval-Valued Intuitionistic Fuzzy Generalized Net of first type (IVIFGN1) if:

a) $A$ is a set of transitions;

b) $\pi_{A}$ is a function giving the priorities of the transitions, i. e.,

$$
\pi_{A}: A \rightarrow N
$$

where $N=\{0,1,2, \ldots\} \cup\{\infty\}$;

c) $\pi_{L}$ is a function giving the priorities of the places, i.e., $\pi_{L}: L \rightarrow N$, where $L=p r_{1} A \cup p r_{2} A$, and $p r_{i} X$ is the $i$-th projection of the $n$-dimensional set, where $n \in N, n \geq 1$ and $1 \leq k \leq n$ (where $L$ is the set of all GN-places);

d) $c$ is a function giving the capacities of the places, i.e., $c: L \rightarrow N$;

e) $f$ is a function which evaluates the truth values of the transition's conditions predicates $r_{i, j}$ in the form $\left\langle M_{i, j}, N_{i, j}\right\rangle$, where $M_{i, j}, N_{i, j} \subseteq[0,1]$ are closed sets, and $\sup M_{i, j}+\sup N_{i, j} \leq 1$.

f) $\theta_{1}$ is a function giving the next time-moment when a transition can become active, i.e., $\theta_{1}(t)=t^{\prime}$, where $t, t^{\prime} \in\left[T, T+t^{*}\right]$ and $t \leq t^{\prime}$. The value of this function is calculated at the moment when a transition terminates its active state;

g) $\theta_{2}$ is a function giving the duration of the active state of a transition, i.e., $\theta_{2}(t)=t^{\prime}$, where $t \in\left[T, T+t^{*}\right]$ and $t^{\prime} \geq 0$. The value of this function is calculated at the moment of activation;

h) $K$ is the set of the GN's tokens.

i) $\pi_{K}$ is a function giving the priorities of the tokens, i.e., $\pi_{K}: K \rightarrow N$;

j) $\theta_{K}$ is a function giving the time-moment when a token can enter the net, i.e., $\theta_{K}(\alpha)=t$, where $\alpha \in K$ and $t \in\left[T, T+t^{*}\right]$;

k) $T$ is the time-moment when the GN starts functioning. This moment is determined with respect to a fixed (global) time-scale;

1) $t^{o}$ is an elementary time-step related to the fixed (global) time-scale;

$\mathrm{m}) t^{*}$ is the duration of the functioning of the net;

n) $X$ is the set of all initial characteristics the tokens can receive when they enter the net;

o) $\Phi$ is a characteristic function which gives a new characteristic to every token when it moves from an input to an output place of a given transition;

p) $b$ is a function giving the maximum number of characteristics a given token can receive, i.e., $b: K \rightarrow N$. 
If for a certain token $\alpha, b(\alpha)=1$, the token will enter the net with an initial characteristic (as a zero-characteristic). After this, it will keep only its current characteristic.

When $b(\alpha)=\infty$, the token $\alpha$ will receive all possible characteristics.

When $b(\alpha)=k<\infty$, except its zero-characteristic, the token $\alpha$ will keep the last $k$ as its characteristics (older characteristics will be "forgotten"). Hence, in general, every token $\alpha$ has $b(\alpha)+1$ characteristics.

In $[4,6,12]$, two algorithms for the tokens transfer are given. Here, we modify them.

The algorithm for tokens transfer after the time moment $t_{1}=T I M E$ (here and below, we denote by TIME the current time moment of the GN), denoted by algorithm A, takes into consideration the possibility of merging and splitting tokens.

A token can be transferred from an input place to a certain output place, even when the capacity of this output place is reached, if the token can be merged with one or more tokens in this output place. This action will not affect the capacity of the output place. The actual number of tokens in the output place will not exceed the maximum number of tokens allowed.

In order for a token $\alpha$ to be merged with one or more tokens $\left\{\beta_{1}, \ldots, \beta_{k}\right\}$ in an output place, these tokens have to be specified in the initial characteristics of the $\alpha$ token.

A symbol $S \in\left\{Y_{S}, N_{S}\right\}$ can be added as a second component of the initial characteristics of the $\alpha$ token. $Y_{S}$ means that the token can split and $N_{S}-$ that it cannot split.

For example, the expression

$$
x_{0}^{\alpha}=“\left\langle\left\{\beta_{1}, \ldots, \beta_{k}\right\}, Y_{S}, x_{0}^{\alpha, *}\right\rangle "
$$

denotes that the $\alpha$ token can split and can be merged with the tokens in the set $\left\{\beta_{1}, \ldots, \beta_{k}\right\}$. The rest of the information of the token's initial characteristics is stored in $x_{0}^{\alpha, *}$.

When the criterion $C n$ for $1 \leq n \leq 15$ is fixed, the algorithm $A$ is described in 12 steps, as follows:

- (A01) Sort the input and output places of the transitions by their priorities.

- (A02) Form two lists of tokens in each input place. The first list contains those of the tokens that can be transferred to a certain output place during the current time moment. Sort these tokens by their priorities. The second list is an empty one at first. These two lists shall be denoted with $P_{1}(l)$ and $P_{2}(l)$, respectively.

- (A03) Generate an empty IM $R$ that corresponds to the IM of the predicates $r$. Assign the value $\langle[0,0],[1,1]\rangle$ to all elements $R_{i, j}$ of $R$ which:

- (A03a) are in a row that corresponds to an empty input place, i.e. there are no tokens in the input place that can be transferred to an output place of the current transition;

- (A03b) are in a column that corresponds to a full output place which no tokens can be transferred to;

- (A03c) are placed in $(i, j)$ place for which the predicate $r_{i, j}$ is set as false or $m_{i, j}=0$, i.e. the current capacity of the arc between the $i$-th input place and the $j$-th output place is 0 . 
Assign the value $\langle[1,1],[0,0]\rangle$ to those elements $R_{i, j}$ of $R$ that are placed in $(i, j)$ place for which the predicate $r_{i, j}$ is set as true.

- (A04) Iterate through the input places in the order set by their priorities, starting with the place with highest priority for which no token has been transferred during the current time step and which has at least one token in it. The token that will be transferred, if possible, is the one with the highest priority in the $P_{1}$ list of the current input place. Perform consequently the following steps in order to determine if and where to transfer the current token.

- (A04a) Check the next $R_{i, j}$ value of $R$. If the value of $R_{i, j}$ has not been set yet, go to step (A04b). If $R_{i, j}=\langle[1,1],[0,0]\rangle$, go to step (A04c). If $R_{i, j}=\langle[0,0],[1,1]\rangle$, check if the corresponding output place has a token that can be merged with the one being transferred. If so, go to step (A04c). Otherwise, go to step (A04d).

- (A04b) Evaluate the truth value of the corresponding predicate $r_{i, j}$ of the IM $r$. If $r_{i, j}$ satisfy criterion $C n$, set the $R_{i, j}$ value of $R$ to $\left\langle M_{i, j}, N_{i, j}\right\rangle$ and go to step (A04c). Otherwise, set the $R_{i, j}$ value to $\langle[0,0],[1,1]\rangle$ and go to step (A04d).

- (A04c) The current token is transferred to the corresponding $j$-th output place and moved to its $P_{2}$ list. The token is merged with specified tokens in the output place, if there are such.

If the transferred token is not merged with other tokens or there are no other tokens left in the new host that the transferred one can be possibly merged with, evaluate the characteristic function of this output place. Assign this value as a characteristic of the transferred token.

If there are tokens in the input places of the current transition that can be merged with the transferred one, it will wait in the output place until these tokens are moved. The new characteristics of the transferred token will be assigned after the token is merged with the last of the suitable tokens in the input places or right at the end of the transition functioning.

Tokens, that have entered an input place of the current transition after its activation, are moved to the $P_{2}$ list of the input place.

- (A04d) If a transfer has been made on the current step and the token cannot split, or all the predicates on the corresponding row are checked, go to step (A05). Otherwise, go to step (A04a).

If the splitting of the current token is not allowed, the evaluation of the predicates stops with the first one that satisfies the criterion $C_{n}$. The token then will be moved to the highest priority output place amongst those the token can be transferred to.

If the splitting of the current token is allowed, then the token is split into as many tokens as the number of the $R_{i, j}$ elements with evaluations satisfying criterion $C_{n}$. These new tokens are transferred to the corresponding output places. The characteristic functions of 
the output places are evaluated. The new characteristics are then assigned to the corresponding tokens upon entering the output places. For difference with the ordinary GNs, the tokens characteristics of the IVIFGN1s contain not only the evaluated values by the respective characteristic functions, but also the the IVIFPs that are evaluations of the respective predicates.

- (A05) If the highest priority token cannot be transferred during the current time step, move the token to the $P_{2}$ list of the input place.

- (A06) Increase by 1 the current number of tokens in each output place to which a token has been transferred if the token has not been merged with any of the other tokens in the host. Do not change the current number of tokens in the output place otherwise.

- (A07) Decrease by 1 the current number of tokens in each input place from which a token has been transferred. If the current number of tokens in such an input place becomes 0 , set to $\langle[0,0],[1,1]\rangle$ all the elements in the corresponding row of the IM $R$.

- (A08) Decrease by 1 the capacities of all the arc through which a token has been transferred. If the current capacity of an arc becomes 0 , assign $\langle[0,0],[1,1]\rangle$ to this element of the IM $R$ that corresponds to the arc.

- (A09) If there are more input places with lower priority from which no token has been transferred to an output place, go to step (A04). Otherwise, go to step (A10).

- (A10) Add $t^{0}$ to the current model time.

- (A11) If the value of the current time is less than or equals $t_{1}+t_{2}$ (the time components of the considered transition), go to (A04). Otherwise, go to step (A12).

- (A12) End of the transition's functioning.

The general algorithm for the GN's functioning, denoted by algorithm $B$, is described next.

The concept of an Abstract Transition (AT) is introduced for the purpose of this algorithm as the union of all GN-transitions that are active at a given time moment.

The algorithm $B$ can be described as follows:

- (B01) Put all $\alpha$ tokens for which $\theta_{K}(\alpha) \leq T$ into the corresponding input places of the net.

- (B02) Construct the GN's AT. Initially it is empty.

- $(\mathbf{B 0 3})$ Check if the value of the current time is less or equal to $T+t^{*}$.

- $(\mathbf{B 0 4})$ If the answer to the question in (B03) is "no", go to step (B12). Otherwise, go to step (B05).

- $(\mathbf{B 0 5})$ Find those transitions for which $t_{1}$ is greater than or equal to the current time. 
- (B06) Check the transitions' types of all transitions determined on step (B05). The method used for the evaluation of the transitions' types is as follows:

- (B06a) replace the names of all places used as variables in the Boolean expression of the transition type with the value 0 , if the corresponding place has no tokens in it at the current moment, and with the value 1, otherwise;

- (B06b) calculate the truth value of the Boolean expression, result of (B06a).

- (B07) Add to the AT those transitions, the transition types of which are evaluated as true on step (B06b).

- (B08) Apply algorithm A over the AT.

- (B09) Remove from the AT those transitions which are inactive at the current time moment.

- (B10) Increase the current time with $t^{0}$.

- (B11) Go to step (B03).

- (B12) End of the GN's functioning.

Obviously, every standard GN is an IVIFGN. By analogy with Theorem 4.1.1, [6], we have proved the following

Theorem 1. The functioning and the results of the work of every IVIFGN can be described by an ordinary GN.

Proof. Let the IVIFGN1 $E$ be given. We shall construct a new, but this time ordinary, GN and we shall prove that both nets function equally. In the proof, we use the validity of Theorem 5.3.1, [4], that gives us possibility to research on the functioning and results of the works of the corresponding transitions in both nets (the given IVIFGN1 $E$ and the ordinary GN $G$ corresponding to it, that we will construct below).

Let the components of IVIFGN1 $E$ be marked by index $E$ and these of GN $G$ - by index $G$.

Let GN $G$ have the same graphical structure as GN $E$ and let all its other components, without the function $f_{G}$ and the characteristic function $\Phi_{G}$, be the same in both nets. For example, the sets $X_{E}$ and $X_{G}$ are equal, i.e., the (equal) tokens in both nets will have equal initial characteristics and the transition condition predicates are also equal in both nets.

Below, we shall construct the new $G$-components.

Let for the real number $x$ :

$$
\operatorname{sg}(x)= \begin{cases}1 & \text { if } x>0 \\ 0 & \text { if } x \leq 0\end{cases}
$$

and

$$
\overline{\operatorname{sg}}(x)= \begin{cases}0 & \text { if } x>0 \\ 1 & \text { if } x \leq 0\end{cases}
$$

Function $f_{G}$ is defined with respect to the choice of criterion $C n$, as follows: 
- in case (C1): $f_{G}\left(r_{i, j}\right)=\operatorname{sg}\left(\inf M_{i, j}\right) \cdot \operatorname{sg}\left(\sup N_{i, j}\right)$;

- in case (C2): $f_{G}\left(r_{i, j}\right)=\operatorname{sg}\left(\sup M_{i, j}\right) \cdot \overline{\operatorname{sg}}\left(\sup N_{i, j}\right)$;

- in case (C3): $\left.f_{G}\left(r_{i, j}\right)=\operatorname{sg}\left(\inf M_{i, j}-\frac{1}{2}\right)\right) \operatorname{sg}\left(\sup N_{i, j}\right)$;

- in case (C4): $f_{G}\left(r_{i, j}\right)=\operatorname{sg}\left(\inf M_{i, j}-\frac{1}{2}\right) \cdot \operatorname{sg}\left(\frac{1}{2}-\sup N_{i, j}\right)$;

- in case (C5): $f_{G}\left(r_{i, j}\right)=\overline{\operatorname{sg}}\left(\frac{1}{2}-\inf M_{i, j}\right) \cdot \overline{\operatorname{sg}}\left(\sup N_{i, j}-\frac{1}{2}\right)$;

- in case (C6): $f_{G}\left(r_{i, j}\right)=\operatorname{sg}\left(\sup M_{i, j}-\frac{1}{2}\right) \cdot \operatorname{sg}\left(\frac{1}{2}-\sup N_{i, j}\right)$;

- in case (C7): $f_{G}\left(r_{i, j}\right)=\overline{\operatorname{sg}}\left(\frac{1}{2}-\sup M_{i, j}\right) \cdot \overline{\operatorname{sg}}\left(\sup N_{i, j}-\frac{1}{2}\right)$;

- in case (C8): $f_{G}\left(r_{i, j}\right)=\operatorname{sg}\left(\inf M_{i, j}-\sup N_{i, j}\right)$;

- in case (C9): $f_{G}\left(r_{i, j}\right)=\overline{\operatorname{sg}}\left(\inf M_{i, j}-\sup N_{i, j}\right)$;

- in case (C10): $f_{G}\left(r_{i, j}\right)=\operatorname{sg}\left(\sup M_{i, j}-\sup N_{i, j}\right)$;

- in case (C11): $f_{G}\left(r_{i, j}\right)=\overline{\operatorname{sg}}\left(\sup M_{i, j}-\sup N_{i, j}\right)$;

- in case (C12): $f_{G}\left(r_{i, j}\right)=\operatorname{sg}\left(\sup M_{i, j}\right)$;

- in case (C13): $f_{G}\left(r_{i, j}\right)=\operatorname{sg}\left(\inf M_{i, j}\right)$;

- in case (C14): $f_{G}\left(r_{i, j}\right)=\operatorname{sg}\left(1-\sup N_{i, j}\right)$;

- in case (C15): $f_{G}\left(r_{i, j}\right)=\operatorname{sg}\left(1-\inf N_{i, j}\right)$.

Therefore, function $f_{G}$ gives as a result value

$$
f_{G}\left(r_{i, j}\right)= \begin{cases}1, & \text { if criterion } C n \text { is satisfied } \\ 0, & \text { otherwise }\end{cases}
$$

Let the characteristic function $\Phi_{G}$ be defined by

$$
\Phi_{G}(\alpha)=“\left\langle\Phi_{E}(\alpha), f_{E}\left(r_{i, j}\right)\right\rangle " .
$$

Thus, we described the new components of the GN G. Now, we must show that both nets function equally.

Let $Z$ and $Z^{*}$ be corresponding transitions in the two nets. We shall show that they function equally. The fact that they will have equal tokens in equal input places if they are transitions from first level in the sense of Theorem 5.3.1, [4], is obvious and by induction we will admit that they have equal tokens with equal current characteristics in equal time moments $t_{1}^{Z}$ and $t_{1}^{Z^{*}}$. Then following the sequentially steps of the Algorithm $A$ for token's transfer and its IVIFGN1's modification, we see that in the case of the IVIFGN $E$ the IM is constructed. 


\begin{tabular}{c|c} 
& $l_{1}^{\prime \prime} \ldots l_{j}^{\prime \prime} \ldots l_{n}^{\prime \prime}$ \\
\hline$l_{1}^{\prime}$ & \\
$\vdots$ & $f_{E}\left(r_{i, j}\right)$ \\
$l_{i}^{\prime}$ & $\left(r_{i, j}-\right.$ predicates $)$ \\
$\vdots$ & $(1 \leq i \leq m, 1 \leq j \leq n)$ \\
$l_{m}^{\prime}$ &
\end{tabular}

where $f_{E}\left(r_{i, j}\right) \in \mathscr{P}([0,1]) \times \mathscr{P}([0,1])$ and for the GN $G-$ the IM is

\begin{tabular}{c|c} 
& $l_{1}^{\prime \prime} \ldots l_{j}^{\prime \prime} \ldots l_{n}^{\prime \prime}$ \\
\hline$l_{1}^{\prime}$ & \\
$\vdots$ & $f_{G}\left(r_{i, j}\right)$ \\
$l_{i}^{\prime}$ & $\left(r_{i, j}-\right.$ predicates $)$ \\
$\vdots$ & $(1 \leq i \leq m, 1 \leq j \leq n)$ \\
$l_{m}^{\prime}$ &
\end{tabular}

where for every set $Y, \mathscr{P}(Y)$ is the set of the subsets of set $Y$.

Therefore, from the above we see that the corresponding elements of both IMs are equal. Thus, the tokens in both transitions $Z$ and $Z^{*}$ will make equal transfers from input to output transition places. There, they will receive similar new characteristics. The tokens from the new net will receive more values, but for the proof of the theorem, it is important that that they will receive all the values which the tokens will receive from the first net.

Hence, both transitions really function equally. This completes the proof.

\section{Interval-valued intuitionistic fuzzy generalized nets of third type}

Here, we introduce the following new

Definition 3. The ordered four-tuple

$$
E=\left\langle\left\langle A, \pi_{A}, \pi_{L}, c, f, \theta_{1}, \theta_{2}\right\rangle,\left\langle K, \pi_{K}, \theta_{K}\right\rangle,\left\langle T, t^{o}, t^{*}\right\rangle,\langle X, \Phi, b\rangle\right\rangle
$$

is called an Interval-Valued Intuitionistic Fuzzy Generalized Net of third type (IVIFGN3) if:

- a) $A$ is a set of transitions defined in Definition 1 ;

- points b) - n) and p) from Definition 2 are valid again; while point o) has the form

- o') $\Phi$ is a characteristic function which gives a new characteristic to every token when it moves from an input to an output place of a given transition and its interval-valued intuitionistic fuzzy evaluation $\left\langle M_{i, j}^{*}, N *_{i, j}\right\rangle$, where $M_{i, j}^{*}, N *_{i, j} \subseteq[0,1]$ and $\sup M_{i, j}^{*}+\sup N *_{i, j} \leq 1$. 
There exist some changes in the first algorithm for the tokens transfer, described in previous Section.

The following assertions hold of IVIFGN3 similarly to IVIFGN1.

Theorem 2. For every IVIFGN3 there exists a standard GN that represents it.

\section{Conclusion}

Different operations and relations are defined in the algebraic aspect of GN theory [4, 6]. These operations have significant practical importance. For example, if two different processes which flow parallelly in time are modelled by two GNs, then by the union operation on these nets a new GN can be constructed to describe the whole process, and it is a union of the two nets. For other types of nets, this is not directly possible. The already defined operators also have an important place in the GN theory.

In the near future, similar operations, relations and operators will be defined for the IVIFGN1s and IVIFGN3s.

In the Second part of the present research, we will introduce two new types of GN-extensions, related to Intuitionistic Fuzzy Generalized Nets of second and fourth types.

Following the ideas from $[9,10,14]$, in future, we will discuss the possible applications of the IVIFGN1s and IVIFGN3s as tools in the Data Mining instruments. Also, different processes, described by GNs will be re-modelled by IVIFGN1s and/or IVIFGN3s and especially in the cases, when the processes flow in uncertainty. For example, in [13], a GN-model of the organization of firefighting process is described. This process flow in conditions of high degree of uncertainty. So, in near future we will re-write the model from [13] by an IVIFGN1.

\section{Acknowledgements}

This work is partially supported by the National Science Fund of Bulgaria under Grants DN16/6 "Integrated approach for modeling of the forest fires spread" and DN02/10 "New Instruments for Knowledge Discovery from Data, and their Modelling."

\section{References}

[1] Alexieva, J., Choy, E., \& Koycheva, E. (2007) Review and bibloigraphy on generalized nets theory and applications. In:-A Survey of Generalized Nets (E. Choy, M. Krawczak, A. Shannon and E. Szmidt, Eds.), Raffles KvB Monograph, 10, 207-301.

[2] Atanassov, K. (1985) The Generalized nets and the other graphical means for modelling. AMSE Review, 2(1), 59-64. 
[3] Atanassov, K. (1987) Generalized index matrices. Comptes rendus de l'Academie Bulgare des Sciences, 40(11), 15-18.

[4] Atanassov, K. (1991) Generalized Nets. World Scientific, Singapore.

[5] Atanassov, K. (1999) Intuitionistic Fuzzy Sets. Springer, Heidelberg.

[6] Atanassov, K. (2007) On Generalized Nets Theory. "Prof. M. Drinov" Academic Publishing House, Sofia.

[7] Atanassov, K. (2012) On Intuitionistic Fuzzy Sets Theory, Springer, Berlin.

[8] Atanassov, K. (2014) Index Matrices: Towards an Augmented Matrix Calculus, Springer, Cham.

[9] Atanassov, K. (2015) Intuitionistic fuzzy logics as tools for evaluation of Data Mining processes, Knowledge-Based Systems, 80, 122-130.

[10] Atanassov, K. (2016) Generalized Nets as a Tool for the Modelling of Data Mining Processes. In :- Innovative Issues in Intelligent Systems (V. Sgurev, R. Yager, J. Kacprzyk, V. Jotsov, Eds.), Springer, Cham, 161-215.

[11] Atanassov K., \& Nikolov, N. (2003) Intuitionistic fuzzy generalized nets: definitions, properties, applications. In :- Systematic Organization of Information in Fuzzy Systems (P. MeloPinto, H.-N. Teodorescu and T. Fukuda, Eds.), IOS Press, Amsterdam, 2003, 161-175.

[12] Atanassov, K., \& Sotirova, E. (2017) Generalized Nets Theory, "Prof. M. Drinov" Academic Publishing House, Sofia (in Bulgarian).

[13] Atanassov, K., Vassilev, P., Georgiev, I., \& Tsakov, H. (2018) Generalized net model of the organization of the firefighting activities when dealing with forest fires, Advances in Neural Networks and Applications 2018 (ANNA'18), September 15-17, 2018, St. St. Konstantin and Elena Resort, Bulgaria, Book of Abstracts, p. 17.

[14] Zoteva, D., \& Krawczak, M. (2017) Generalized Nets as a Tool for the Modelling of Data Mining Processes. A Survey. Issues in Intuitionistic Fuzzy Sets and Generalized Nets, 13, $1-60$. 